\title{
CHARACTERIZATION OF FIREFLY, AN IMAGING SPECTROMETER DESIGNED FOR AIRBORNE MEASUREMENTS OF SOLAR-INDUCED FLUORESCENCE
}

\author{
Bruce Cook ${ }^{1}$, Lawrence Corp ${ }^{2}$, Peter Clemens ${ }^{3}$, Ian Paynter ${ }^{4}$, Jyoteshwar Nagol ${ }^{5}$, Joel McCorkel ${ }^{1}$ \\ ${ }^{1}$ NASA Goddard Space Flight Center; ${ }^{2}$ Science Systems and Applications, Inc.; ${ }^{3}$ Headwall Photonics, Inc.; \\ ${ }^{4}$ Universities Space Research Association; ${ }^{5}$ University of Maryland.
}

\begin{abstract}
FIREFLY (Fluorescence Imaging of REd and Far-red Light Yield) is a compact, fine-resolution imaging spectrometer that was designed and assembled by Headwall Photonics (Fitchburg, MA, USA) in collaboration with NASA scientists for airborne measurements of Solar-Induced Fluorescence (SIF). FIREFLY is integrated into the next generation of NASA Goddard's Lidar, Hyperspectral and Thermal airborne imager (G-LiHT; www.gliht.nasa.gov), providing a complete system for measuring, interpreting and scaling SIF emissions. Characterization of FIREFLY was performed here to evaluate its performance and suitability for retrieving SIF.
\end{abstract}

Index Terms- G-LiHT, GLAMR, Imaging Spectroscopy, SIF

\section{INTRODUCTION}

Solar-Induced Fluorescence (SIF) is the re-emittance of solar energy absorbed by plants when light absorption exceeds the utilization capacity of photosynthesis [2], and is directly related to plant health, productivity and light-use efficiency. Measuring SIF has been used to provide quantitative estimates of Gross Primary Production [2], species composition, and phenology and physiological status, including plant stress [2,3] and crop health [4]. Ecological attributes and processes that can be inferred from SIF measurements are of interest at landscape to global scales, and recent successes have been made retrieving SIF from satellites such as GOSAT, GOME-2, SCIAMACHY and OCO-2 $[2,4,5]$.

In addition to SIF retrieval capabilities offered by FIREFLY, coincident LiDAR, hyperspectral, and thermal sensor data from the G-LiHT instrument package provide ancillary information on vegetation structure, pigment concentrations, and non-fluorescent quenching that is required for modeling and interpretation of canopy-level observations of SIF. Understanding the relationship between SIF retrievals, vegetation structure and canopy illumination using fine-resolution $(1 \mathrm{~m}) \mathrm{G}$-LiHT data is critical to scaling and interpreting moderate-resolution $(300 \mathrm{~m})$ data from ESA's FLuorescence EXplorer (FLEX) satellite mission.

SIF is a relatively weak signal [6], reported as approximately $1 \%$ of total absorbed solar energy $[2,3,4], 2 \%$ by some [4], and $1-5 \%$ in the near infrared region [5]. As a result, the accuracy of its retrieval from the solar continuum is greatly influenced by sensor characteristics such as spectral resolution; stray light performance; systematic noise; sensor stability; and detector linearity [2]. Here, we quantify instrument characteristics of FIREFLY to evaluate effects on SIF retrievals using solar Fraunhofer lines.

\section{FACILITIES AND EQUIPMENT}

The next generation G-LiHT airborne imaging system [1] offers many upgrades over its predecessor, including dual Riegl VQ480i lidars; enhanced VNIR hyperspectral imaging with a Headwall Photonics Micro-Hyperspec E-series spectrometer; greater spatial-resolution thermal imaging with a Xenics Gobi 640 camera; fine-resolution (3 cm GSD) RGB stereo images with a Phase One iXU1000-R camera; and enhanced positional accuracies with an Applanix POS AV V6 GPS-INS. System integration and test flights with GLiHT and FIREFLY were completed during February 2017 on a Beechcraft King Air A90.

The FIREFLY imaging spectrometer is an aberrationcorrected, concentric, all-reflective Offner design with a telecentric $25 \mathrm{~mm} \mathrm{f} / 2.5$ lens. The instrument was designed to cover the full range of chlorophyll fluorescence, 670 to 780 $\mathrm{nm}$ at $<0.2 \mathrm{FWHM}$, and offer high performance in a compact, light-weight form. An example of FIREFLY's high fidelity imagery can be seen in Figure 1, with system specifications summarized in Table 1 and a sample vegetation spectra shown in Figure 2. The spectrometer is now commercially available, and additional details can be found on the company website (http://www.headwallphotonics.com/).

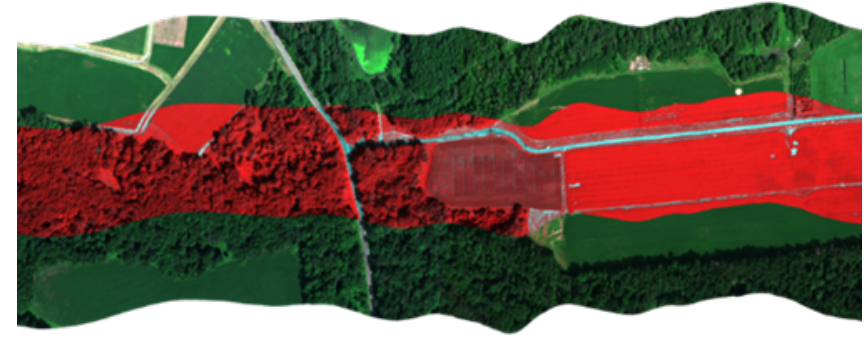

Figure 1. FIREFLY CIR image overlaid on the RGB band combination from G-LiHT's VNIR spectrometer, showing high spatial fidelity of coincident imaging spectrometer data. 


\begin{tabular}{|c|c|c|}
\hline \multirow{4}{*}{ Spectral } & Wavelength Range & 670 to $780 \mathrm{~nm}$ \\
\hline & Bands & 2160 \\
\hline & Sampling Interval & $0.051 \mathrm{~nm}$ \\
\hline & Nominal FWHM & $<0.2 \mathrm{~nm}$ \\
\hline \multirow{7}{*}{ Spatial } & G-LiHT Flight Altitude & $335 \mathrm{~m}$ \\
\hline & Native Spatial Pixels & 1600 \\
\hline & Field of View & $23.5 \mathrm{deg}$ \\
\hline & Swath Width & $140 \mathrm{~m}$ \\
\hline & Instantaneous Field of View & $0.142 \mathrm{deg}$ \\
\hline & Along Track Sampling Interval & $0.84 \mathrm{~m}$ \\
\hline & Integration time & $26.6 \mathrm{~ms}$ \\
\hline \multirow{4}{*}{ Sensor } & FPA Type & TE-cooled sCMOS \\
\hline & Dynamic Range & 16 bit \\
\hline & Pixel Size & $6.5 \mu \mathrm{m}$ \\
\hline & Fullwell Capacity & 30,000 e- \\
\hline
\end{tabular}

Table 1. Key specifications for FIREFLY SN:001 of the Chlorophyll Fluorescence Imaging Spectrometer series.

Calibration of G-LiHT's optical sensors is performed annually using NASA's Goddard Laser for Absolute Measurement of Radiance (GLAMR; glamr.gsfc.nasa.gov) facility. GLAMR is NIST traceable through the use of transfer radiometers, and owes its heritage to the Spectral Irradiance and Radiance responsivity Calibrations using Universal Sources (SIRCUS) system [7]. GLAMR consists of a wavelength-tunable laser source fiber coupled to an integrating sphere providing finely tunable monochromatic light (Fig. 3). A full aperture absolute calibration approach allows determination of the absolute spectral responsivity (ASR) of an instrument at any given wavelength [8] (Fig. 4) traceable to NIST's Primary Optical Watt Radiometer (POWR) through transfer radiometers [9].

The GLAMR facilities instrument was used to measure the FIREFLY imaging spectrometer over its wavelength response range from $690 \mathrm{~nm}$ to $780 \mathrm{~nm}$. Four ranges were sampled with fine $0.05 \mathrm{~nm}$ steps, and six ranges were sampled at a courser resolution of $0.15 \mathrm{~nm}$ steps. The full wavelength range and beyond to $1000 \mathrm{~nm}$ was sampled at 1 $\mathrm{nm}$ steps to investigate the potential for spectral stray light from within and beyond the spectral range of the sensor.
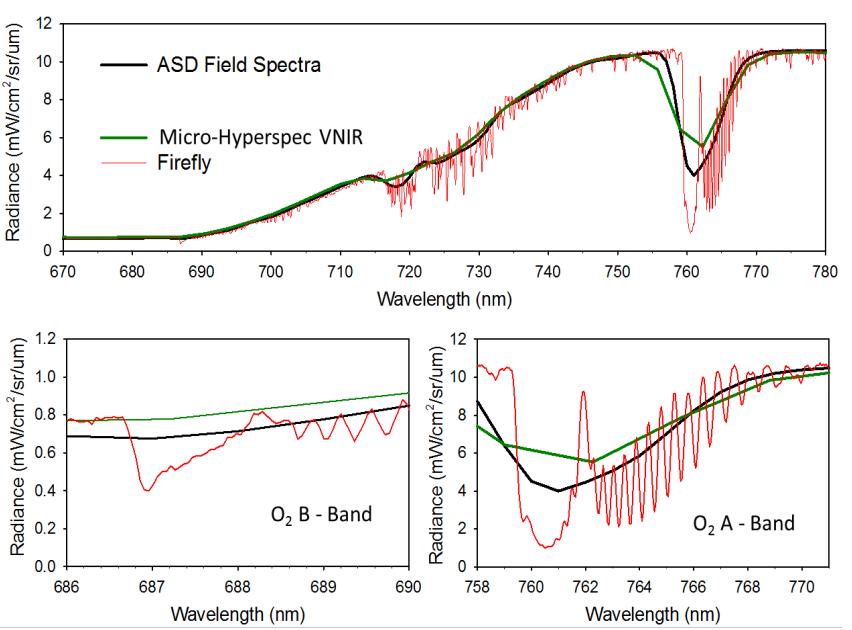

Figure 2. Example vegetation radiance spectra from FIREFLY and G-LiHT's VNIR imaging spectrometers.

Spatial uniformity and more frequent assessments of radiometric stability of G-LiHT's optical sensors is performed periodically during the year with the Labsphere Helios D-Series uniform source. A combination of three NIST traceable Tungsten lamps and a variable aperture allows for a wide dynamic range in radiometric output used to accurately characterize sensor system linearity and SNR response functions. Further, wavelength stability assessments of G-LiHT's optical systems are periodically performed by coupling a set of $\mathrm{Ar}, \mathrm{Kr}, \mathrm{Xe}, \mathrm{Ne}$ and $\mathrm{Hg}$ pen style calibration lamps to the accessory port of the Helios sphere. Any shift in the reported location of these narrow emission lines would indicate instability of the corresponding optical system.

\section{RESULTS}

GLAMR radiance measurements were combined with the dark current adjusted DNs, to provide Absolute Spectral Response (ASR), DN per ( $\left.\mathrm{W} \mathrm{m}^{-2} \mathrm{~nm}^{-1} \mathrm{sr}^{-1}\right)$, for each spectral pixel, and were derived through equations and procedures detailed by Barnes et al., 2015 [9]. ASRs were interpolated
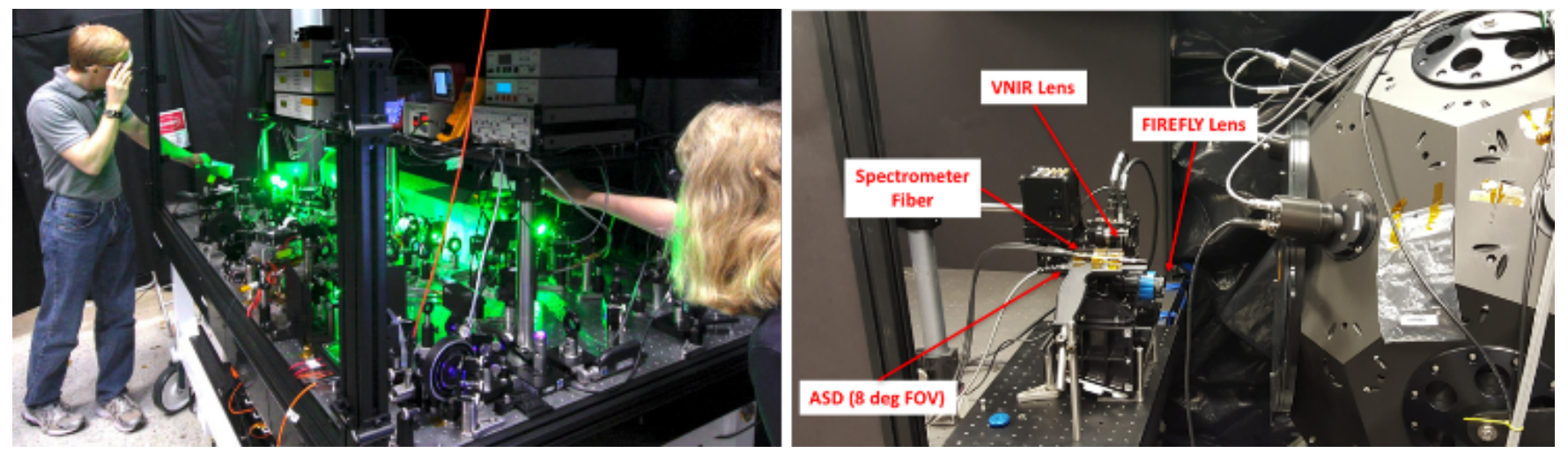

Figure 3. NASA's Goddard Laser for Absolute Measurement of Radiance (GLAMR) being configured for absolute radiometric response characterization of G-LiHT's FIREFLY and VNIR imaging spectrometers. 

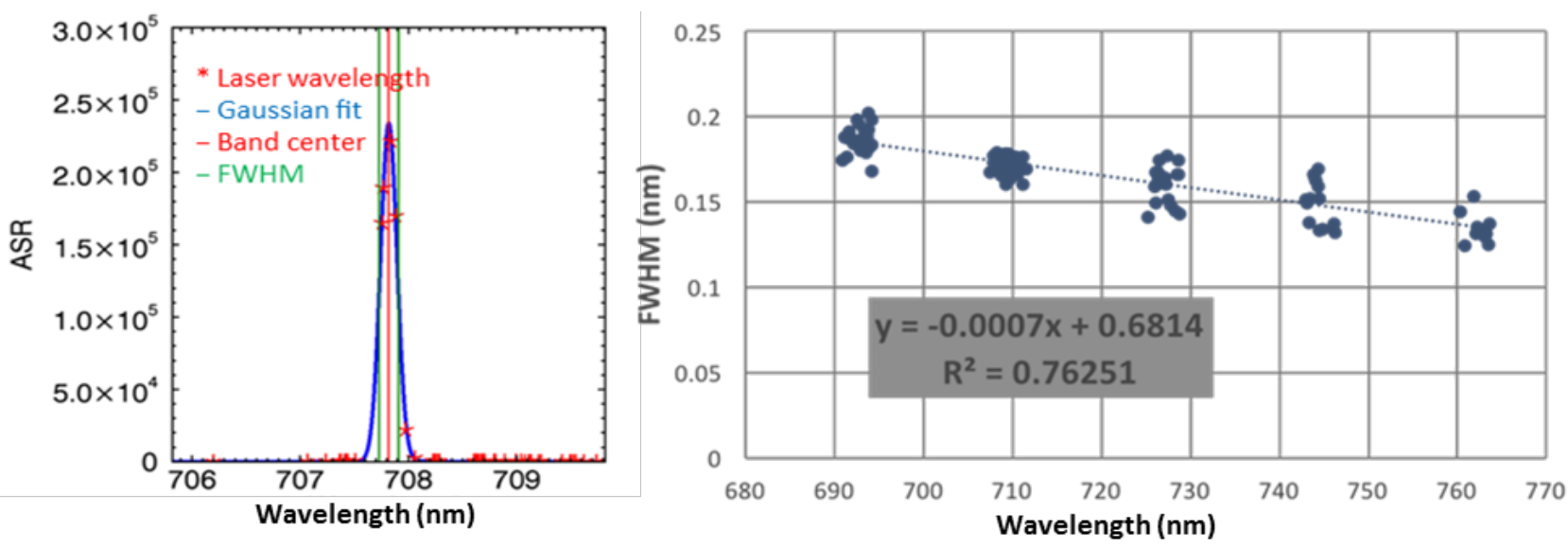

Figure 4. Sample Absolute Spectral Response (ASR) function for a single pixel of the Firefly focal plane (left) and the Full Width Half Maximum (FWHM) spectral resolution of the sensor system as characterized using GLAMR (right).

to the nearest $0.001 \mathrm{~nm}$ and fit with a Gaussian function for a total of 104 spectral bands.

Spectral stray light was described by a spectral stray light Signal Distribution Function (SDF) described by Zong et al. [10]. The ASR for each band (Fig. 4) was used to generate the SDF matrix for each of FIREFLY's spatial cross track pixels. SDF quantifies the magnitude of the out-of-band response when compared to the in-band response. From these data, a spectral stray light correction matrix was derived that allows for correction of very low levels of stray light emanating primarily from the shoulders of the spectral response functions. The influence of applying this stray light correction on SIF retrievals is being investigated. The FWHM spectral resolution for the FIREFLY spectrometer was also derived from the ASRs and varied between 0.20 and 0.12 in the red and far-red parts of the spectrum, respectively.

The Helios Uniform Source was used for characterization of FIREFLY's detector linearity and Signal to Noise performance (SNR). Illumination intensity of the source was varied in fine steps covering the entire dynamic range of the FIREFLY sensor. FIREFLY SNR performance was calculated as the mean over the standard deviation of 1000 repeated observations for the $740 \mathrm{~nm}$ spectral band (Fig. 5, left). The linearity response was determined by normalizing signal DN values to the maximum DN signal accumulation rate (Fig. 5, right). A $7^{\text {th }}$ order polynomial regression was then used to determine the linearity response function. FIREFLY's focal plane array was determined to have a highly linear response range from $5 \mathrm{~K}$ to $40 \mathrm{~K} \mathrm{DN}$ with nonlinearities less than $0.4 \%$. The nonlinearity compensation increases rapidly as DN values depart from this range.

\section{DISCUSSION}

Further characterization of G-LiHT is planned for February 2018 to extend spectral range covered for ASR's and spectral stray light assessments to include wavelengths shorter than $690 \mathrm{~nm}$. Additional fine spectral sampling across the FIREFLY instrument range will be acquired and assessed, as will measurements of instrument stability. The monochromatic tunable laser system will also be used to further verify the systems linearity response functions by sampling discrete wavelengths and finely adjusting the laser output through a variable attenuator. GLAMR's linearized NIST traceable transfer radiometers and monochromatic laser source will be used to validate measurements with the Helios Uniform Source.

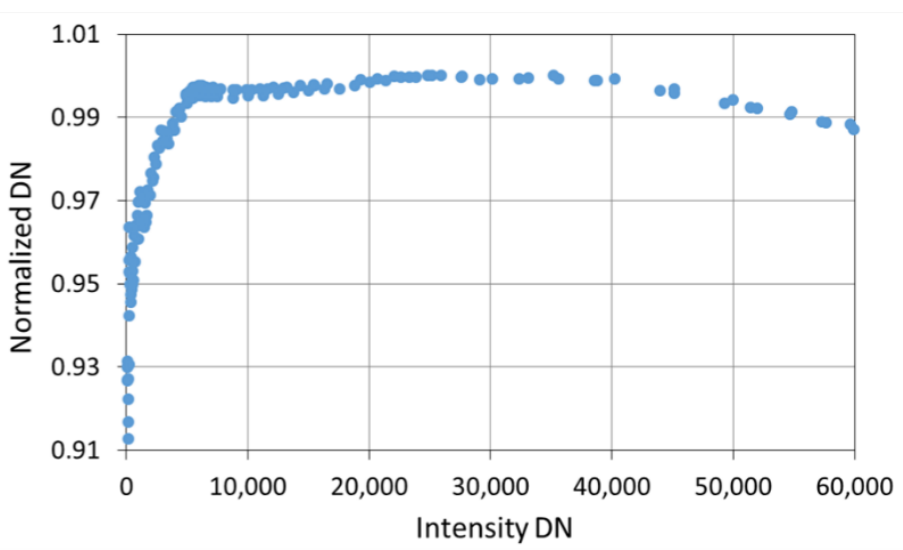

Figure 5. FIREFLY SNR (left) and linearity (right) functions as determined using the Helios D-Series Uniform Source. 
Following integration into the G-LiHT system, FIREFLY has been used to acquire greater than 300 hours of airborne data acquisition covering a wide range of biomes, including tropical forests; mid-latitude broadleaf deciduous and needleleaf forests; boreal forests; wetlands; and croplands. FIREFLY has proven to be quite robust and has not exhibited systematic variation from its initial calibration state. The temperature stabilized focal plane enabled operation of the sensor system even as aircraft cabin temperature exceeded $40^{\circ} \mathrm{C}$ without adverse impact on dark current or systematic noise. High altitude unpressurized transits and operation in humid environments also did not affect system performance.

FIREFLY is capable of high spatial resolution observations which could provide tree-scale measurements of SIF, and possibly even intra-canopy variations. However, spatial aggregation may be needed to reduce retrieval errors associated with instrument characteristics (e.g., SNR) and environmental conditions (e.g., solar irradiance).

\section{CONCLUSIONS}

Herein we provided an initial characterization of the FIREFLY (Fluorescence Imaging of REd and Far-red Light Yield) high performance spectrometer for retrieving estimates of Solar Induced Fluorescence (SIF). FIREFLY is integrated into NASA Goddard's Lidar, Hyperspectral and Thermal (G-LiHT) airborne instrument package to provide a complete system for measuring, interpreting and scaling SIF emissions. FIREFLY's performance was characterized with NASA's Goddard Laser for Absolute Measurement of Radiance (GLAMR) facility, which provided data needed to ensure the retrieval of SIF using Fraunhofer Line Depth (FLD) methods for solar features outside the $\mathrm{O}_{2} \mathrm{~A}$-band (756 to 759,770 to $775 \mathrm{~nm}$ ), thereby avoiding complications due to $\mathrm{T}, \mathrm{P}, \mathrm{O}_{2}, \mathrm{H}_{2} \mathrm{O}$ and cloud and aerosol particulates. Very low-level spectral stray light was observed and is correctable. Signal to Noise Ratio (SNR) calculated over the instruments dynamic range suggested that fluorescence levels of $1 \%$ would be observable by FIREFLY under normal illumination conditions, with the potential to further reduce errors by spatial aggregation of fine-resolution $(1 \mathrm{~m})$ image data.

New, high-fidelity airborne imaging spectrometers such as FIREFLY are able to provide landscape-scale observations of SIF at fine spatial resolutions and temporal frequencies that are needed to scale and interpret both ground- and satellite-based measurements. Well characterized, high performance spectrometers for SIF retrieval are particularly relevant to the upcoming ESA Fluorescence Explorer (FLEX) mission, by providing calibration and validation data and contributing to the science-based interpretation of the SIF signal. Coincident acquisition with multi-sensor data from NASA's G-LiHT system provides additional ancillary information (e.g., vegetation indices related to chlorophyll content and non-photochemical quenching; absorbed PAR; canopy cover and rugosity; leaf area; and canopy surface temperature) that will improve our understanding and interpretation of the SIF signal, particularly in forests with complex canopy structure, variable illumination conditions and shadowing.

\section{REFERENCES}

[1] Cook, B.D., L. A. Corp, Nelson, R.F., Middleton, E.M., Morton, D.C., McCorkel, J.T., Masek, J.G., Ranson, K.J., Ly, V. and Montesano, P.M., 2013. NASA Goddard's LiDAR, hyperspectral and thermal (G-LiHT) airborne imager. Remote Sensing, 5(8), pp.4045-4066.

[2] Frankenberg, C., Fisher, J.B., Worden, J., Badgley, G., Saatchi, S.S., Lee, J.E., Toon, G.C., Butz, A., Jung, M., Kuze, A. and Yokota, T., 2011. New global observations of the terrestrial carbon cycle from GOSAT: Patterns of plant fluorescence with gross primary productivity. Geophysical Research Letters, 38(17).

[3] Jeong, S.J., Schimel, D., Frankenberg, C., Drewry, D.T., Fisher, J.B., Verma, M., Berry, J.A., Lee, J.E. and Joiner, J., 2017. Application of satellite solar-induced chlorophyll fluorescence to understanding large-scale variations in vegetation phenology and function over northern high latitude forests. Remote Sensing of Environment, 190, pp.178-187.

[4] Guanter, L., Frankenberg, C., Dudhia, A., Lewis, P.E., GómezDans, J., Kuze, A., Suto, H. and Grainger, R.G., 2012. Retrieval and global assessment of terrestrial chlorophyll fluorescence from GOSAT space measurements. Remote Sensing of Environment, 121, pp.236-251.

[5] Joiner, J., Yoshida, Y., Vasilkov, A.P., Corp, L. A. and Middleton, E.M., 2011. First observations of global and seasonal terrestrial chlorophyll fluorescence from space. Biogeosciences, 8(3), pp.637-651.

[6] Alonso, L., Gomez-Chova, L., Vila-Frances, J., Amoros-Lopez, J., Guanter, L., Calpe, J. and Moreno, J., 2008. Improved Fraunhofer Line Discrimination method for vegetation fluorescence quantification. IEEE Geoscience and Remote Sensing Letters, 5(4), pp.620-624

[7] Brown, S.W., Eppeldauer, G.P. and Lykke, K.R., 2006. Facility for spectral irradiance and radiance responsivity calibrations using uniform sources. Applied Optics, 45(32), pp.8218-8237.

[8] Barnes, R.A., Brown, S.W., Lykke, K.R., Guenther, B., Butler, J.J., Schwarting, T., Turpie, K., Moyer, D., DeLuccia, F. and Moeller, C., 2015. Comparison of two methodologies for calibrating satellite instruments in the visible and near-infrared. Applied optics, 54(35), pp.10376-10396.

[9] McCorkel, J., Thome, K., Hair, J., McAndrew, B., Jennings, D., Rabin, D., Daw, A. and Lunsford, A., 2012, August. Instrumentation and first results of the reflected solar demonstration system for the Climate Absolute Radiance and Refractivity Observatory. In Proc. of SPIE Vol (Vol. 8510, pp. 85100B-1).

[10] Zong, Y., Brown, S.W., Lykke, K.R. and Ohno, Y., 2007, July. Correction of stray light in spectroradiometers and imaging instruments. In CIE (Vol. 178, No. D2, pp. 33-36). 